\title{
La institucionalidad de la gestión de riesgo a desastres en Guatemala
}

\author{
María Carlota Ávila Arbizú \\ Licenciada en Trabajo Social y Maestra en Educación y Ambientalización Curricular \\ Estudiante del Doctorado en Investigación para el Desarrollo Social \\ Centro Universitario de Sur Oriente, CUNSURORI \\ Universidad de San Carlos de Guatemala
}

Fecha de recepción: 28/05/2018 Fecha de aceptación: 30/07/2018

\begin{abstract}
Resumen
La incorporación de la temática sobre la gestión de riesgos en Guatemala, ha cobrado auge después de la emergencia de eventos naturales que marcaron la escasa capacidad de respuesta en el país. Con el enfoque de riesgo se puede visibilizar los factores y contextos adversos a nivel local, municipal, departamental y nacional que contribuyen a la construcción de escenarios y medidas de prevención ante eventos adversos. A partir de las condiciones propias de cada comunidad, dentro del país se debe construir la gestión de riesgos que habrá de asumir características, estrategias e instrumentos particulares de acuerdo a las condiciones propias de cada lugar; aspectos que deben ser considerados en la institucionalidad del riesgo.
\end{abstract}

\section{Palabras clave}

Desarrollo social, institucionalidad, gestión de riesgos, descentralización.

\begin{abstract}
The incorporation of the theme on risk management in Guatemala, has gained momentum after the emergence of natural events that marked the poor response capacity in the country. With the risk approach, the factors and adverse contexts at the local, municipal, departmental and national levels that contribute to the construction of scenarios and preventive measures against adverse events can be made visible. Based on the conditions of each community, within the country, risk management must be built, which must assume specific characteristics, strategies and instruments according to the conditions of each place; aspects that should be considered in the risk institutionality.
\end{abstract}

\section{Keyword}

Social development, institutionality, risk management, descentralization. 


\section{Introducción}

Pensar el desarrollo social, implica la conjugación de diversos actores y escenarios diversos, en donde la gestión local del riesgo es un aspecto intrínseco; adscrito al desarrollo imperante como un factor de sostenibilidad que permite la permanencia del modelo y las medidas de prevención, mitigación y respuesta adecuada ante los embates de los desastres.

La incorporación de la temática de gestión de riesgo a desastres a la escena nacional ha cobrado auge a partir de la creación de la Coordinadora Nacional para la Reducción de Desastres (CONRED). La creación de la Política Nacional para la reducción de riesgo a desastres, operativiza en las instancias nacional, departamental y municipal la capacidad de respuesta ante un evento adverso.

La descentralización de la CONRED a través de las delegaciones departamentales permite el abordaje desde lo local de las principales amenazas que se registran en la población, lo que a su vez admite revisar los indicadores para verificar si las acciones emanan de las necesidades propias de la población. La implementación de la política evidencia la institucionalidad de la gestión del riesgo a desastres en el país y los desafíos que aún se presentan en el marco de su fortalecimiento y del crecimiento demográfico y los embates del cambio climático en Guatemala.

\section{Problema}

La vulnerabilidad a desastres naturales en el país se aborda con mayor auge a partir de la formulación de la Política Nacional de Gestión de Riesgo a Desastres. Se pretende realizar una aproximación al análisis del proceso de institucionalidad de la gestión de riesgo a desastres y el panorama en torno a su aplicabilidad.

\section{Metodología}

La metodología utilizada corresponde a dos procesos: análisis retrospectivo de la incorporación de la gestión de riesgo a desastres en el escenario nacional y el análisis de la situación actual de la institucionalidad de la temática en el país, especificando en la implementación de la política sectorial.

En relación con el análisis de la institucionalidad de la gestión de riesgos, se realizará una aproximación a las características que manifiestan Braun y Velez (2004) respecto a la política institucionalizada, los cuales hacen referencia a: la existencia de una estrategia a largo plazo, la continuidad de actores, la existencia de espacios de coordinación, el desarrollo de la capacidad técnica, la existencia de mecanismos de recolección y análisis de información confiable y la participación de actores sociales.

\section{Marco teórico}

Definir el desarrollo es una tarea compleja, vinculada a la marcha histórica de la sociedad y su evolución, las diferentes acepciones que se ubican para el término han sido objeto de procesos que emergen de la relación de los actores implicados. Cada uno de los modelos de desarrollo instaurados adquieren matices particulares que los distinguen uno de otro y cuya aplicación se vincula con las condiciones previas e histórico sociales de los países en donde se instaura. Pensar en el desarroIlo social, implica la conjugación de diversos actores y escenarios sociales que han sido objeto de análisis a nivel internacional.

Se han celebrado dos cumbres mundiales sobre desarrollo social en Copenhague (1995) y Ginebra (2000), derivados de las mismas resultan los acuerdos ratificados por los países miembros de la Organización de Naciones Unidas (ONU), en donde se establece la relación entre la responsabilidad de las instituciones tanto públicas como sociales para 
facilitar las inversiones productivas a fin de satisfacer las necesidades y capacidades de las personas, en este ámbito definir el desarrollo social es una tarea indispensable. En la Cumbre Mundial de Desarrollo Social (1995) en el punto 7 se establece que:

[...] el desarrollo social es un elemento fundamental de las necesidades y aspiraciones de las personas del mundo entero y de las responsabilidades de los gobiernos y de todos los sectores de la sociedad civil. Declaramos que, en términos económicos y sociales, las políticas y las inversiones más productivas son las que facultan a las personas para aprovechar al máximo sus capacidades, sus recursos y sus oportunidades. Reconocemos que no se puede lograr un desarrollo social y económico sostenible sin la participación de la mujer y que la igualdad y la equidad entre la mujer y el hombre constituye una prioridad para la comunidad internacional $y$, como tal, debe ser un elemento fundamental del desarrollo económico y social (ONU, 1995).

Estas cumbres han delineado las acciones latinoamericanas para abordar el problema del desarrollo:

La crisis de los enfoques de desarrollo prevalecientes en la región: se evidencia en las presiones acuciantes para acentuar la mercantilización de los territorios y su incorporación a las dinámicas de la acumulación financiera del capitalismo. De ahí que se observe la tendencia a la ampliación de la frontera extractivista en sus diferentes modalidades: a) ampliación de la frontera petrolera, con énfasis ahora en la exploración y explotación de petróleo en mar abierto, glaciales, reservas naturales y territorios indígenas, b) la industrialización de bituminosas y de esquisto, c) la minería a cielo abierto, d) el agronegocio, que incluye pesticidas, transgénicos, monocultivos y sistemas de plantación, e) los servicios ambientales (privatización de agua, mercados de carbono, industria del turismo, pago por servicios ambientales, y f) la biotecnología, la geotecnología, y los biocombustibles, entre otros (Albuja \& Dávalos, 2012)
Desde el punto de vista epistemológico, los modelos de desarrollo primario exportador, de industrialización por sustitución de importaciones y el neoliberal, pese a sus importantes diferencias, poseen rasgos comunes en los que sobresalen:

a) la concepción de la modernización como un proceso evolutivo y lineal basado en el crecimiento económico como factor fundamental,

b) la subordinación y negación del valor intrínseco de la naturaleza en las relaciones del ser humano con el entorno, $y$

c) la subordinación de los saberes subalternos a la racionalidad occidental dominante.

Los diferentes modelos de desarrollo que se han establecido en Latinoamérica han influido en el diseño e implantación de las políticas públicas de Guatemala y direccionado la gestión pública y el gasto social a la apropiación de modelos y estrategias materializadas en las políticas sociales. La Constitución Política de la República de Guatemala establece en el artículo 119 que son obligaciones del Estado: a) promover el desarrollo económico de la nación; b) velar por la elevación del nivel de vida de todos los habitantes, procurando el bienestar de la familia; y en general, c) promover las condiciones necesarias que potencien el desarrollo económico y social de la población, con especial interés en aquellos grupos que se encuentran en situación de pobreza y pobreza extrema.

En ese sentido, se han generado las condiciones necesarias para que el desarrollo económico de la nación se operativice a través de las políticas. Es preciso recalcar que a partir de la apertura democrática en 1985 el modelo de desarrollo imperante en el país y las políticas que han emergido han cambiado el rumbo de la sociedad guatemalteca en función de direccionar el país hacia las políticas de ajuste estructural generados por el neoliberalismo.

El neoliberalismo no ha presentado un éxito arrasador. La privatización ha sido utilizada para la realización de negocios en las altas esferas, no está ga- 
rantizado el crecimiento y tampoco la estabilidad y los efectos sociales en términos de la pobreza han sido significativos, lo que ha contribuido al debilitamiento de las políticas sociales, restringiendo el acceso de los grupos pobres a los servicios básicos, la concentración de oportunidades del mercado se encuentran en pocos grupos; por lo que no se tiene claridad en las ventajas del mercado que pareciera que cada vez más amplía la brecha de la desigualdad social en el país.

El proceso de descongestión se expresa a partir de la formulación de la Política Nacional de descentralización (Decreto 14-2002), la cual se contempla ser un medio para la reducción de la pobreza y la consolidación de la democracia, a través de la oportuna prestación de servicios en los departamentos y municipios de la nación, incorpora el concepto de equidad de género como clave para el desarrollo humano sostenible y el acceso y control de los recursos.

La descentralización es el proceso mediante el cual se transfiere desde el Organismo Ejecutivo a las municipalidades y demás instituciones del Estado y a las comunidades organizadas legalmente, con participación de las municipalidades, el poder de decisión, la titularidad de la competencia, las funciones, los recursos de financiamiento para la aplicación de las políticas públicas nacionales, a través de la implementación de políticas municipales y locales en el marco de la más amplia participación de los ciudadanos y ciudadanas. La descentralización es un proceso de carácter político, que demanda la distribución territorial del poder público entre entidades políticas dotadas de autonomía y de gobiernos propios electos libremente.

Se incorporan a la escena nacional la temática sobre ordenamiento territorial y el desarrollo sostenible. Al respecto, se desarrolla por la Secretaría de Planificación y Programación de la Presidencia (SEGEPLAN) los Planes de Desarrollo Municipal (PDM), para todos los municipios del país, enfatizando que el ordenamiento territorial es un proceso técnico, administrativo y político, en el cual como punto de partida es de vital importancia la toma de decisiones concertadas con los actores involucrados, pues lo que se pretende es considerar las condiciones sociales, ambientales y económicas para la ocupación de forma ordenada del territorio, así como el aprovechamiento eficiente de los recursos naturales, lo cual busca el desarrollo equilibrado de las poblaciones en forma sostenible, disminuyendo los impactos negativos que puedan ocasionar las diferentes actividades y procesos de desarrollo del municipio, esto con el objeto de mejorar la inversión pública.

En este mismo ámbito, en la parte considerativa de la Ley de Consejos de Desarrollo Urbano y Rural (Decreto 11-2002) se establece que es esencial que el Sistema de Consejos de Desarrollo se rija por los principios de igualdad y dignidad en derechos de todos los actores sociales, haciéndose efectivos estos derechos en condiciones de oportunidades equitativas de participación, dentro de una convivencia pacífica, en el marco de una democracia funcional, efectiva y participativa, en los procesos de toma de decisión en la planificación y ejecución de las políticas públicas de desarrollo. Por otra parte, define responsabilidades, en cada uno de los niveles de la gestión pública: nacional, regional, departamental, municipal y comunitario, con el objeto de formular las políticas, planes, programas y proyectos de desarrollo, según sean los ámbitos correspondientes.

Se persigue a través de esta iniciativa establecer un orden adecuado en el manejo del gasto público y la inversión social de acuerdo a los escenarios priorizados y en el marco del alcance de los Objetivos de Desarrollo del Milenio ODM, los cuales pretenden a partir de ocho objetivos encausar las acciones de desarrollo impulsadas por los países miembros de la ONU hacia el alcance de las metas del milenio para el 2015. Cabe señalar que los informes al respecto al finalizar el plazo del tiempo no fueron alentadores. Los enunciados del objetivo 7 y 8 se encaminaron hacia asegurar un ambiente sano y saludable y lograr una sociedad global para el desarrollo; en este sentido el tema del desarrollo sostenible encuentra eco a nivel internacional y nacional. 
Como parte del seguimiento que se realiza a la agenda 2015, se establecen los Objetivos de Desarrollo Sostenible (ODS), con metas propuestas para el 2030 y en donde se persigue que los países sean colocados en la senda del desarrollo incluyente, sostenible y resiliente. La Agenda 2030 cuenta con 17 objetivos para poner fin a la pobreza, luchar contra la desigualdad y la injusticia y hacer frente al cambio climático. Este marco es tomado como referencia para la elaboración del plan de desarrollo de Guatemala.

Dentro del Plan de Desarrollo K'atun 2032 se encuentra contemplado el apartado 8 Recursos naturales hoy para el futuro, en donde se establece que "La sostenibilidad ambiental constituye uno de los pilares fundamentales del desarrollo nacional y exige actuaciones sociales, económicas y políticas inmediatas y sostenidas frente a los actuales niveles de degradación. Uno de los retos es la comprensión de la integralidad en el abordaje de las prioridades ambientales, así como la consolidación de un sistema que articule a los actores clave con los diversos factores que rodean dicha integralidad" (CONADUR/SEGEPLAN, 2014).

Dicha declaración encamina los esfuerzos institucionales hacia el alcance de los ODS estableciendo una estrecha relación en la planificación para su alcance. Como objetivo del eje se encuentra: Proteger y potenciar los recursos naturales en equilibrio con el desarrollo social, cultural, económico y territorial, para que permitan satisfacer las demandas actuales y futuras de la población en condiciones de sostenibilidad y resiliencia, ante el impacto de los fenómenos que la naturaleza presente.

En este marco se considera pertinente la incorporación de la temática de la Gestión Local del Riesgo, pues se supone que la planificación del desarrollo es vinculante con la reducción y control del riesgo de desastres como una estrategia para su sostenibilidad, además cada modelo de desarrollo implica en sí mismo la construcción de riesgos sociales.

La Ley de Desarrollo Social en el artículo 22 referente a Población, ambiente y recursos naturales establece:
ARTíCULO 22. Población, ambiente y recursos naturales. El Estado, por medio del Ministerio de Ambiente y Recursos Naturales, Ministerio de Economía, Ministerio de Trabajo y Previsión Social, y la Secretaría de Planificación y Programación de la Presidencia, efectuará evaluaciones de impacto sobre el ambiente y estudios e investigaciones sobre los vínculos, efectos e impactos existentes entre la población y consumo, producción, ambiente y recursos naturales, que sirvan de orientación para realizar acciones dirigidas al desarrollo sostenible y sustentable (Congreso de la República de Guatemala, 2001).

El desarrollo implica la inclusión de escenarios sociales en los que se encuentra vinculado el Estado y las instituciones públicas que administra en la gestión pública. Los actores sociales que se vinculan en la implantación de los modelos de desarrollo actúan conforme a las políticas públicas emanadas del gobierno; estas a su vez responden a los convenios y ratificaciones internacionales que el país ha desarrollado y que influencian la gobernanza.

La gestión del riesgo hace referencia a un proceso social y político a través del cual la sociedad busca controlar los procesos de creación o construcción de riesgo o disminuir el riesgo existente con la intención de fortalecer los procesos de desarrollo sostenible y la seguridad integral de la población. Es una dimensión de la gestión del desarrollo y de su institucionalidad (Lavell, 2006).

La política institucional constituye un andamiaje que a partir de un acuerdo político y social atiende un problema específico, posee una estructura claramente definida que se rige por los lineamientos establecidos por el país para su formulación e implantación, por lo que requiere de la incorporación de actores ligados a su operatividad; en Guatemala se registran políticas públicas, sociales y sectoriales.

Por lo que al referirse a las secretarías y comisiones de Estado en Guatemala, a estas les concierne impulsar las políticas sectoriales del país. De tal forma que a la CONRED le corresponde establecer los mecanismos y normas para la reducción de 
desastres mediante la coordinación interinstitucional; dicho marco resultó el escenario propicio para iniciar en el 2006 los esfuerzos por la formulación de la Política Nacional para la Reducción de Desastres, que fue aprobada en Acta 03-2011 según Acuerdo 06-2011 del Congreso de la República de Guatemala.

Dicha política, es el resultado del trabajo interinstitucional realizado por varias instituciones y organizaciones, tanto públicas como privadas, que coordinadas por la Secretaría Ejecutiva de CONRED, unieron esfuerzos para dar seguimiento a lo establecido en una de las estrategias del Programa Nacional de Prevención y Mitigación ante Desastres 2009-2011, que platea la formulación de la política y en donde se establece su operatividad a nivel nacional, regional, municipal y local; esta última instancia como eslabón para vincular la temática directamente con las poblaciones a nivel municipal.

El desarrollo municipal es un proceso social y político reflejo del desempeño de la gestión pública y los actores económicos y comunitarios, la gestión del riesgo es también reflejo de este desempeño, en forma de acciones integradas en los diferentes temas e instrumentos del desarrollo municipal; acciones que comprenden el conocimiento y manejo del riesgo, así como de manejo del desastre.

La gestión del riesgo de desastres no es tarea exclusiva de los gestores de situaciones de desastre, sino que debe implicar desde los ciudadanos, líderes políticos, instituciones gubernamentales, sector privado, organizaciones de la sociedad civil, asociaciones profesionales y organismos científicos y técnicos; de tal forma que se empodere a todos los sectores para tomar decisiones que contribuyan a reducir el riesgo.

La reducción del riesgo de desastres debe presentar una sólida base institucional para la implementación de las medidas necesarias que permitan su institucionalización en los diversos escenarios sociales. La incorporación de procesos de educación colectivos permitirán la creación de una cultura de seguridad y resistencia en todos los niveles, lo que contribuirá a la reducción de riesgos subyacentes y la capacidad de respuesta y recuperación a nivel local, regional y nacional.

La gestión de riesgo busca recuperar, valorar, sistematizar, hacer expresas las acciones y estrategias aisladas que se han desarrollado en las comunidades en su afán de adaptar a las exigencias de un medio ambiente en permanente transformación, el resultado de la sistematización permite que los actores sociales puedan de forma concertada, planificada y consciente encaminar a la comunidad hacia el desarrollo sostenible la gestión del desarrollo sostenible. Incluye sí temas como la prevención de amenazas (cuando ello sea posible), la mitigación de la vulnerabilidad, la atención a los desastres (preparación, atención a las emergencias y rehabilitación) y las actividades de reconstrucción física y recuperación de ecosistemas y comunidades afectadas por un desastre, pero va más allá. La gestión del riesgo más que algo que tenga que ver específicamente con los desastres, es un tema que tiene que ver con el desarrollo (Wilches Chaux, 1998)

Wilches Chaux (1996) define la sostenibilidad como la capacidad de un sistema o proceso (en este caso el sistema comunidad-ambiente), para cumplir el objetivo o propósito colectivo de las interacciones entre sus elementos o actores, y para transformarse y evolucionar cuantitativa y cualitativamente, sin poner en peligro las bases o fundamentos de los cuales depende la permanencia en el largo plazo de ese mismo sistema o proceso.

Según Cardona (1993) un desastre se puede definir como un acontecimiento social que se desencadena como resultado de la ocurrencia de un evento que puede ser de origen natural o antrópico. Este trastorno sobre las condiciones normales de funcionamiento de una comunidad, le impide la ejecución de las actividades que involucra su vida diaria, y provoca pérdidas de bienes y en ciertas ocasiones vidas humanas, requiriendo de una respuesta para atender a los afectados y restablecer las condiciones habituales de la comunidad. Visto desde esta perspectiva "los desastres no son naturales". 
Con respecto al tema relacionado con la vinculación entre desastres y desarrollo han surgido enfoques como el conocido del comportamiento que plantea que los desastres dependen de un evento geofísico (Hewill, 1983), este planteamiento se centra en el estudio de los eventos naturales que disparan desastres, y en las soluciones estructurales requeridas para mitigar el desastre, visto así las comunidades son ajenas a la ocurrencia de desastres, y las personas se limitan a seguir instrucciones de expertos en lo que respecta a su comportamiento ante una situación de riesgo.

Por lo que se considera que la vinculación de la gestión del riesgo se encuentra estrechamente ligada a los modelos de desarrollo imperantes en el país y que establecen en la actualidad un vínculo estrecho entre el ordenamiento territorial y el desarrollo sostenible.

La concepción del riesgo y la terminología asociada a su definición no sólo ha variado con el tiempo, sino también desde la perspectiva disciplinaria desde la cual se ha abordado. Esto significa que actualmente no existe una concepción que unifique las diferentes aproximaciones o que recoja de manera consistente y coherente los diferentes enfoques (Darío Cardona, 2001), sin embargo se considera que la gestión de riesgo es el proceso de adopción de políticas, estrategias y prácticas orientadas a evitar y reducir los riesgos de desastres o minimizar sus efectos (PDRS-GTZ-DGPM-MEF 2009) (Chuquisengo, 2011).

Lavell, refiere que la gestión de riesgo tendrá sus características, estrategias e instrumentos particulares, por lo que debe ser considerada en su esencia, un componente intrínseco y esencial de la gestión social. En este sentido, todo proceso de desarrollo de transformación territorial y ambiental debe ser formado por un sistema de análisis y control de los factores de riesgo existentes o posibles.

\section{Resultados}

\subsection{Panorama histórico respecto a la gestión de riesgos en el país}

El terremoto del 76 en Guatemala dejó al descubierto la debilidad estructural de la infraestructura del país y la debilidad en la capacidad de respuesta institucional ante el embate de la naturaleza; aspectos que influyeron en la aparición de asentamientos en la periferia de la ciudad y en la respuesta institucionalizada para la emergencia en el país.

Los impactos del huracán Mitch, 1998, provocaron en un sector de la sociedad civil de Centroaméri$\mathrm{ca}$, la necesidad de plantear la gestión del riesgo como un nuevo paradigma en la concepción y prevención del desastre, lo cual implicó una profunda reflexión y cuestionamiento a los procesos de ayuda de emergencia, rehabilitación y reconstrucción, pero sobre todo al modelo de desarrollo. Dichos eventos favorecieron la revisión de la capacidad de respuesta en Guatemala y volvieron a hacer evidente la necesidad de contar con una institución rectora de la gestión de riesgos en el país. A partir de lo cual se originó lo realización de los esfuerzos para la creación de la Política Nacional para la Reducción de Riesgo a Desastres en Guatemala.

La aprobación de la Política Nacional para la Reducción de Riesgo a Desastres en Guatemala en Acta 03-2011, según Acuerdo 06-2011 del Consejo Nacional de CONRED en Guatemala, cumplió con lo establecido en la prioridad No.1 del Marco de Acción de Hyogo que se refiere a que la reducción de riesgos es una prioridad nacional y local.

Dicha política está diseñada en forma transversal, lo que permite a los sectores público, privado, sociedad civil y cooperación internacional asumir roles para institucionalizar y fortalecer una cultura de prevención de desastres y resiliencia en Guatemala. Es preciso señalar que existen instituciones que han trabajado la gestión de riesgos en diferentes momentos. 


\section{Cuadro 1 \\ Instituciones que han trabajado la Gestión de Riesgos en Guatemala}

Institución

Conformación del Foro para la Gestión de Riesgos que luego cambió su nombre por Concertación Regional para la Gestión de Riesgos

El Centro de Coordinación para la Prevención de los Desastres Naturales en América Central (CEPREDENAC)

Secretaría Ejecutiva de la Coordinadora Encargada de coordinar los aspectos referentes a la Coordinadora Nacional de Reducción de Desastres. Nacional para la Reducción de Desastres en los 22 departamentos del Guatemala (SE-CONRED)

\section{COCIGER}

Oficina de la Pastoral Social del Arzobispado de Guatemala (OPSAG)

\section{SEGEPLAN}

Ministerio de Educación

Universidad de San Carlos de Guatemala

Universidad Rafael Landívar

\section{Principales funciones}

Creación de la Convergencia Ciudadana para la Gestión de Riesgos en Guatemala. Establecida en noviembre de 2002, con fuerte apoyo de la Pastoral Social de Guatemala y la Fundación Técnica en Proyectos (FUNDATEP), ambas también contrapartes de OXFAM América

Ente especializado en protección contra riesgos y desastres designado por los gobiernos centroamericanos en el marco del Sistema de Integración Centroamericana (SICA). Está integrado por una Secretaría Ejecutiva y seis comisiones nacionales encabezadas por el organismo nacional de protección civil de cada país país

Ocasionalmente tiene contactos institucionales con la CONRED en el nivel central para el desarrollo de encuentros, diálogos y participación en capacitaciones. A partir de 2010 se estableció una relación de trabajo continuo y sistemático.

A partir de 2002 empezaron a trabajar el área de Gestión de Riesgos a nivel comunitario

Incorpora el enfoque de la gestión del riesgo en la inversión en el diseño de los proyectos de inversión pública en las etapas de preinversión, inversión y operación

Incorporan la temática de gestión de riesgos a la enseñanza dentro de las instituciones educativas de los diferentes niveles en función a los lineamientos establecidos por la CONRED en sus directrices generales para la construcción de Planes Escolares de Respuesta

Incorporación de la temática de Gestión de Riesgos en algunas carreras, creación de comisión respecto al tema

Cooperación con instancias internacionales para el apoyo en programas nacionales sobre gestión de riesgos

Fuente: Elaboración propia

La incorporación de la temática de gestión de riesgos a nivel institucional ha permitido un avance en su abordaje e incorporación a la programación de las instituciones en la planificación de sus activida- des anuales. Evidencia de ello es que en el 2002 la Oficina de Pastoral Social del Arzobispado de Guatemala (OPSAG); incorpora el área de gestión de riesgo a sus procesos de trabajo. 


\section{Cuadro 2 \\ Características del Área de Gestión de Riesgo trabajadas por OPSAG}

\section{Objetivo}

Contribuir en los diversos procesos orientados a la prevención y mitigación de desastres en las comunidades más vulnerables de la Arquidiócesis de Guatemala (Departamentos de Guatemala y Sacatepéquez), apoyando las distintas acciones de formación en materia de Organización Comunitaria, autogestión e incidencia que se realiza coordinadamente con las áreas de la OPSAG, con las agencias gubernamentales y organizaciones no gubernamentales a nivel nacional

\section{Estrategias}

Diagnosticar y censar comunida- $\sqrt{ }$ des en riesgo

$\sqrt{ }$ Generar procesos y brindar herramientas a agentes de pastoral, $\sqrt{ }$ líderes comunitarios y voluntarios para prevenir y reducir desastres

$\sqrt{ }$ Apoyar el proceso de organización en las comunidades en riesgo

$\sqrt{ }$ Crear espacios de gestión, organización, respuesta solidaria y atención urgente

$\sqrt{ } \quad$ Ubicar y facilitar albergues

$\sqrt{ }$ Orientar y acompañar la gestión comunitaria

$\sqrt{ } \quad$ Establecer coordinación interinstitucional en el tema de Gestión del Riesgo

$\sqrt{ } \quad$ Realizar procesos formativos a través de la capacitación en gestión de riesgo

$\sqrt{ }$ Brindar asesoría y orientación sobre el tema

$\sqrt{ }$ Acreditar las organizaciones locales

$\sqrt{ }$ Apoyo a través de diversos tipos de material educativo

\section{Actividades estratégicas}

Formar y fortalecer los grupos parroquiales organizados en acciones de acompañamiento y asesoría

$\sqrt{ }$ Realizar procesos de formación y capacitación de los agentes de pastoral en cultura de prevención y gestión de riesgo

$\sqrt{ }$ Fomentar el voluntariado juvenil y comunitario en las áreas urbano marginales y rurales

$\sqrt{ } \quad$ Potenciar el liderazgo con valores en la Doctrina Social de la Iglesia

$\sqrt{ }$ Apoyar formas organizativas que faciliten la incidencia de la política partiendo del nivel local hacia otros niveles

Fuente: Elaboración propia con base a documento de sistematización de las lecciones aprendidas del riesgo de OPASG 2002

Cabe señalar que los proyectos desarrollados por OPSAG, se realizaron a nivel comunitario en los departamentos de Sacatepéquez y Guatemala, todos trabajados a nivel comunitario, en los municipios, cantones y asentamientos. La cobertura que se ubicó para la realización de los mismos constituyó zonas priorizadas respecto al alto índice de vulnerabilidad detectado.

\subsection{Análisis de la institucionalidad de la gestión de riesgos}

Se realiza una aproximación al análisis de la institucionalidad de la gestión de riesgo en Guatemala, para lo cual se tomó como referencia el plantea- miento realizado por Braun \& Velez (2004) quienes indican que una política institucionalizada se caracteriza por: la existencia de una estrategia a largo plazo, la continuidad de actores, la existencia de espacios de coordinación, el desarrollo de la capacidad técnica, la existencia de mecanismos de recolección y análisis de información confiable y la participación de actores sociales.

En función de lo cual se considerará la institucionalidad de la política como aquella que emana de un acuerdo político y social para atender un problema en específico, que establece objetivos y metas claras, y cuenta con instrumentos para evaluar su consecución, que establece obligaciones y derechos específicos para cada uno de los actores relevantes 
y que cuenta con definición de normas y reglas de comportamiento (Eisenstadt, 1979).

\subsubsection{La existencia de una estrategia a largo plazo}

La estrategia planteada a largo plazo, corresponde a la Ley de la Coordinadora para la Reducción de Desastres (Decreto 106-96), que legalmente es reconocida por el Congreso de la República de Guatemala, de donde emanaron los esfuerzos para la formulación de la Politica de Gestión de Riesgo a Desastres, cuya intervención a nivel nacional se basó desde 2005 a finales de 2015 en la prioridad de acción específica No.1 del Marco de Acción de Hyogo "Garantizar que la reducción del riesgo de desastres sea un prioridad nacional y local dotada con una sólida base institucional para su implementación" (CONRED, 2011 pág. 3).
A partir de este marco internacional se construyó un marco nacional para la reducción de riesgo de desastres, en donde se promovió la realización de planes y actividades en todos los niveles administrativos, desde el nivel nacional hasta el local. Se generaron guías para la elaboración de los planes a nivel departamental y municipal, los cuales fueron coordinados a través de los delegados departamentales al interior del país.

La incorporación de la gestión de riesgo a desastres se ha extendido a la inversión pública, en donde se incorporó la variable de riesgo en las diferentes fases del ciclo del proyecto. El análisis de riesgo en proyectos de inversión pública AGRIP, se incorpora desde el Sistema Nacional de Planificación de Desarrollo.

Cuadro 3

Enfoque de la gestión del riesgo en la inversión

\section{Fases}

$\begin{array}{ll} & \text { Idea } \\ & \text { Perfil } \\ \frac{c}{\frac{0}{2}} & \text { Prefactibilidad } \\ \frac{\frac{1}{d}}{0} & \text { Factibilidad } \\ & \text { Diseños finales }\end{array}$

Etapas

\section{Gestión del riesgo}

Análisis de amenazas

Por zona

Mapas de amenazas

Amenazas

Análisis de vulnerabilidad

Del sitio (exposición)

Por fragilidad

Por resiliencia

Identificación de medidas de reducción de riesgos

$$
\frac{c}{\frac{1}{0}}
$$

Ejecución

Implementación de medidas para la reducción del riesgo

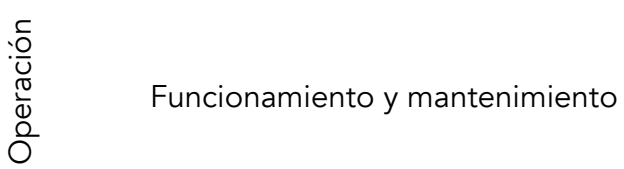

Monitoreo y seguimiento de la implementación de las medidas de reducción de riesgo

Evaluación ex-post

Fuente: SEGEPLAN 2009 pág. 19. 
En los proyectos de inversión pública la gestión del riesgo tiene un enfoque integral que se aplica a lo largo de todas las fases y etapas del ciclo del proyecto: preinversión, inversión y operación. (SEGEPLAN, 2013 pág.11).

Se prevé un ordenamiento de la gestión del riesgo en la preparación y presentación de proyectos que deben ser incorporados en el diagnóstico, formulación y evaluación, lógicamente deben llenar los requisitos estipulados para su aprobación. La programación de la inversión pública anualmente incorpora la cartera de proyectos a ejecutar y la inclusión de la gestión del riesgo representa una estrategia a largo plazo.

En la actualidad, se respalda el quehacer en materia de gestión de riesgos en la III Conferencia Mundial de Naciones Unidas en Sendai, en donde se adoptó el Marco Sendai para la reducción del riesgo a desastres 2015-2030 como sucesor del Marco de Acción de Hyogo. La nueva era es la gestión integral de reducción del riesgo a desastres basados en: i) la inclinación a la respuesta, ii) el enfoque integral (incorporando la gobernanza e inclusión) y iii) la vinculación con las agendas de reducción de la pobreza, desarrollo y cambio climático y el fomento de la resiliencia. Lo que evidencia la directriz de una estrategia a largo plazo para el abordaje de la temática.

\subsubsection{La continuidad de actores}

La continuidad de actores se encuentra representada al interior de la República según lo establecido en la Ley de la Coordinadora Nacional para la Reducción de Desastres (CONRED), que en su Artículo 9, hace referencia a los niveles de operación dentro de la institución a través de la Coordinadora Departamental (CODRED), Coordinadora Municipal (COMRED) y Coordinadora Local para la Reducción de Desastres (COLRED). A dichas instancias se les asigna un rol específico de acuerdo a las funciones propias de cada coordinadora, destacando el principio de centralización normativa y descentralización ejecutiva.

La continuidad de los actores permanece únicamente en los delegados departamentales, que para fines del desarrollo del trabajo se encuentran limitados a cubrir las atribuciones asignadas en cuanto que cubren lo relacionado a aspectos técnicos y secretariales de cada departamento. En el caso de la continuidad de actores en la SEGEPLAN cada delegación cuenta con personal permanente que da seguimiento a los requerimientos de presentación de proyectos.

En ambos casos no existe discontinuidad en los programas que se ejecutan dentro de las instituciones, al contrario existen mejoras a los planteamientos realizados y actualizaciones de acuerdo a la ratificación de acuerdos internacionales por parte del país.

\subsubsection{La existencia de espacios de coordinación}

La coordinación interinstitucional se realiza en cada uno de los niveles de intervención de la CONRED. En el caso de las delegaciones departamentales estas tienen a su cargo la coordinación con las instancias a nivel departamental, municipal y local. Conviene señalar, que en la mayoría de las casos en cada departamento del país únicamente se encuentra contratado un profesional encargado de la oficina; aspecto que limita el funcionamiento y aumenta las actividades que debe desarrollar de acuerdo a las funciones que se le asignan dentro de la institución.

Sin embargo, la coordinación entre instituciones gubernamentales y no gubernamentales se evidencia en el involucramiento de sus representantes en los diferentes programas y actividades que se realizan. En el caso de la delegación de Jalapa, se han establecido alianzas estratégicas para potencializar las acciones encaminadas a la implantación de la gestión de riesgo. Se han desarrollado alianzas institucionales a través de las cuales se han desarrollado tres Planes de Respuesta Municipal, específicamente para los municipios de Jalapa, San Pedro Pinula y San Luis Jilotepeque, en donde se coordinó con nueve instituciones y se planificó respecto a 30 amenazas identificadas. 


\subsubsection{El desarrollo de la capacidad técnica}

La capacidad técnica de los delegados de la CONRED es fortalecido a través de procesos de actualización respecto a la temática, lo que permite que las funciones ejercidas se realicen en apego a lo que indica la teoría y se aplique en respuesta a las necesidades locales. Al mismo tiempo se realizan actividades educativas en las cuales se educa a la población respecto a la gestión de riesgo a desastres. Mismos procesos desarrolla SEGEPLAN en las delegaciones departamentales a través de seminarios/taller a los representantes de la Oficina Municipal de Planificación de las diferentes municipalidades. A estos ultimos se les capacita respecto al Análisis de Gestión de Reisgo en Proyectos de Inversión Pública (AGRIP).

\subsubsection{La existencia de mecanismos de recolección y análisis de información confiable}

Existen estrategias de recolección y medición de datos, de acuerdo a indicadores determinados por la CONRED a nivel nacional. Estos permiten la toma de decisiones, evaluación y seguimiento de las acciones a tomar dentro de la institución.

El enfoque de gestión del riesgo en la inversión pública contempla su propia metodología enfocada en: i) la identificación y priorización de amenazas, ii) análisis de la vulnerabilidad. La gestión del riesgo consiste en la "planificación y aplicación de medidas orientadas a impedir o reducir los efectos adversos de los fenómenos peligrosos sobre la población, los bienes, los servicios y el ambiente" (SEGEPLAN, 2013 pág. 30), para el cumplimiento se realizan las siguientes actividades:

$>$ Identificación de medidas de reducción del riesgo.

> Estimación de costos y beneficios por opción.

$>$ Evaluación y selección de la mejor opción (SEGEPLAN, 2006).
Cada proyecto debe contar con un reporte de análisis de gestión del riesgo en la inversión pública, para que le sea asignado un código en el Sistema Nacional de Inversión Pública (SNIP). El nuevo paradigma sustituye el enfoque emergencista de atención del desastre, el cual es relevante porque se incorpora desde el Sistema Nacional de Planificación de Desarrollo.

\subsubsection{La participación de actores sociales}

El involucramiento de los actores sociales es indispensable en cuanto permite que se consolide la continuidad de las políticas y los programas emanados de ellas. El establecimiento de convenios interinstitucionales y la red de voluntariado permite la incorporación de mejoras a programas. En el caso de los voluntariados y los programas de educación escolarizada y no formal permiten que la población se interese por la temática y que se apropien de ella.

\section{Conclusiones}

Los recurrentes embates de los desastres en el país han generado reflexión y acción respecto al abordaje del problema del riesgo a desastres. Esto ha permitido el avance en la institucionalidad de la gestión de riesgos a nivel de gobierno, sociedad civil y cooperación. A pesar de ser un tema que causa divisiones en su abordaje, las características de vulnerabilidad propias del país lo hacen parte de la agenda consensuada de estos tres actores (gobierno, sociedad civil y cooperación/organizaciones no gubernamentales).

Si bien, existe una institucionalidad definida por lineamientos en cuanto a la organización y funcionamiento de la gestión del riesgo en Guatemala, a través de los cuales se delimitan los mecanismos de participación y gobernabilidad, estos requieren un compromiso gubernamental para garantizar el despliegue de las instituciones de gobierno a fin de incrementar la cobertura y promover el involucramiento de los líderes locales en la toma de decisiones y acciones que contribuyan a crear una cultura de resiliencia en el país. 


\section{Bibliografía}

Coordinadora Nacional para la Reducción de Desastres, CONRED. (2011). Política nacional para la reducción de riesgo a los desastres en Guatemala. Guatemala: CONRED.

Agudelo, C. (1999). Desarrollo y salud. Revista de salud pública, I(1), 1-18.

Albuja, V., \& Dávalos, P. (2012). Extractivismo posneoliberalismo: El caso de Ecuador. Recuperado el 10 de octubre de 2017, de estudiosdeldesarrollo.net/critical/rev4/3,pdf

Armijo, M. (2011). Planificación estratégica e indicadores de desempeño en el sector público. Santiago de Chile: Insituto Latinoamericano y del Caribe de Planificación Económica y Social ILPES.

Becker, E. (s.f.). Transformación ecológica social: Notas para una ecología política sostenible. Recuperado el 14 de octubre de 2017, de file:///C:/Users/Avila/Downloads/abri041\%20 (2).pdf

Braun, M., \& Velez, C. (2004). Retos institucionales para la equidad y sustentabilidad del desarroIlo Social en América Latina. Wachington DC: Presentado en el IV Foro de Equidad social .

Cálix R., Á. (2016). Los enfoques de desarrollo en América Latina: Hacia una transformación social-ecológica. Análisis(1), 34.

Cardona, A. (1993). Economía, salud-enfermedad y modelos de desarrollo. Bogotá, Colombia: Instituto Coloombiano de Antropología y Cultura.

Chávez Gutiérrez, M. R. (s.f.). Los enfoques del desarrollo social: Las perspectivas del bienestar en México. México.

CONADUR/SEGEPLAN. (2014). Plan nacional de desarrollo K'atun: Nuestra Guatemala 2032. Guatemala: Autor.
Congreso de la República de Guatemala. (2001). Ley de Desarrollo Social: Decreto 42-2001. Guatemala: Autor.

Consejo Nacional de Desarrollo Urbano y Rural, CONADUR. (2014). Plan Nacional de DesarroIlo K'atun: Nuestra Guatemala 2032. Guatemala: CONADUR/SEGEPLAN.

Darío Cardona, O. (2001). La necesidad de repensar de manera holística los conceptos de vulnerabilidad, riesgo: Una crítica y una revisión necesaria para la gestión. Bogotá, Colombia: Centro de estudios sobre Desastres y Riesgos, CEDERI, Universidad de Los Andes.

Eisenstadt, S. (1979). Enciclopedia internacional de las ciencias sociales (Vol. IV). España: Aguilar.

Franco , R., \& Pardo, M. (2010). Institucionalidad social en América Latina. Santiago de Chile: CEPAL.

Guillén, A. (2007). Modelos de desarrollo y estrategias alternativas en América Latina. Recuperado el 15 de octubre de 2017, de www. centrocelsofurtado.org.br/arquivos/ image/201108311505340.A_GUILLEN3.pdf

Lavell, A. (2006). Consideraciones en torno al enfoque, los conceptos y los términos que rifen con referencia a la reducción del riesgo y la atención de desastres en los países Andinos miembros del CAPRADE. Colombia: PREDECAN.

Mejía-Ortega, L. M., \& Franco-Giraldo, Á. (2007). Protección social y modelos de desarrollo en América Latina. Salud Pública, 9(3), 471-483.

Miner Fuentes, Y. (2002). Determinación de riesgo: análisis y generación de mapas. Informe final. Guatemala: Consultora Proyecto "Reducción del Riesgo Asociado a Desastres Naturales en Asentamientos del área metropolitana de Guatemala". 
Molina Jiménez, C. (2002). Ética y educación. Guatemala: MINEDUC.

Munch, L. (2008). Planeación estratégica: El rumbo hacia el éxito. México: Trillas.

Navarro Restrepo, A. (s.f.). Elementos de planeación estratégica: Metodología y ejemplo desarrollado.

ONU, Organización de las Naciones Unidas. (1995). Cumbre mundial sobre desarrollo social. Dinamarca: Autor.

Pérez Sánchez, A. (2015). Finanzas y economía. Recuperado el 4 de octubre de 2017, de http:// www.finanzzas.com/industrializacion-por-sustitucion-de-importaciones-isi

Pérez Ventura, J. (2015). Introducción al concepto de desarrollo. Recuperado el 15 de octubre de 2017, de http:// elordenmundial.com/2015/01/09/ introduccion-al-concepto-de-desarrollo/

Presidencia de la República de Uruguay. (2005). Guía metodológica de planificación estratégica. Uruguay: Oficina de Planeamiento y Presupuesto.
Secretaría de Planificación y Programación de la Presidencia, SEGEPLAN. (2013). Análisis de gestión del riesgo en proyectos de inversión pública. Guatemala: Autor.

Secretaría de Planificación y Programación de la Presidencia, SEGEPLAN. (2013). Análisis de gestión del riesgo en proyectos de inversión pública-AGRIP-. Guatemala: Autor.

SEGEPLAN. (2006). Informe fortalecimiento de capacidades para la reducción de riesgos en los procesos de desarrollo, Consultoría de Francisco Mendoza. Guatemala: Autor.

Wilches Chaux, G. (1996). Introducción al concepto de sostenibilidad global. Santa Fé de Bogotá, Colombia.

Wilches Chaux, G. (1998). Auge, caída y levantada de Felipe Pinillo, mecánico y soldador o yo voy a correr el riesgo: Guía de la red para la gestión local del riesgo. Perú: La Red. 